\title{
INTELLIGENT MODELLING WITH ALTERNATIVE APPROACH: APPLICATION OF ADVANCED ARTIFICIAL INTELLIGENCE INTO TRAFFIC MANAGEMENT
}

The currently existing transport infrastructures are failing due to many problems. This paper deals with presenting a new approach of modelling and forecasting transport processes using artificial intelligence. Firstly, the current state of forecasting transport data is presented; the traditional as well as new artificial intelligence methods, such as artificial neural networks, are discussed and described. After that, a support vector regression prediction model is briefly presented and an empirical analysis is performed. Finally, on the basis of our experiment and performed comparative analysis we state that artificial intelligence (AI) intelligent methods have potential in the transport area as they can improve the efficiency, safety, and environmental compatibility of transport systems.

Keywords: forecasting, artificial intelligence, intelligent transport system, machine learning, support vector machines, support vector regression, $R$ software, statistical methods

\section{Introduction}

The main goal of the transport system is to ensure safe, efficient and reliable transportation while minimizing its negative impact on the environment. However, existing transport infrastructures are failing due to many problems, such as capacity problems, poor safety, unreliability, environmental pollution and inefficiency.

In recent years, artificial intelligence (AI) has attracted big attention of researchers in many different branches from signal processing, pattern recognition, travel time estimations [1], rail vehicle system [2] or time series forecasting [3]. Also in transport, there is a possibility for using artificial intelligence techniques to contribute to the development of new, intelligent modes of operation for existing infrastructures. $\mathrm{AI}$ is already deployed in many areas of transport, e.g. intersection control on arterial roads, travel time predictions and vehicle fuel injection systems [4].

Transport problems exhibit several features that allow application of methods and tools of artificial intelligence. Transport systems can often be very difficult to be simulated using the traditional approach, mainly because of interactions between different elements of the transport system. When dealing with transport problems, one often must solve difficult optimisation problems that cannot be fully met by using traditional mathematical programming methods. Artificial intelligence provides a wide range of tools and methods for a solution of transport problems. Methods and tools of artificial intelligence are primarily used to predict the behaviour of transport systems, transport optimisation problems in control systems and clustering, also in the transport planning process, decision making and pattern recognition [5].

Artificial intelligence applications are very efficient on extremely big data sets (gigabytes of data) where classical statistical or econometrical methods could not be used because of computational or time (cost efficiency) reasons [6].

AI techniques are applied in dynamic traffic management including evolutionary algorithms, knowledge-based systems, neural networks and multiagent systems. According to Miles and Walker [4], it is possible to distinguish between:

- direct control (measures using traffic lights, 'smart' barriers and variable message signs (VMS) to allocate traffic priorities in time and space) and

- Indirect control measures like recommendations for drivers which focus on the behaviour of individual vehicles (e.g. radio broadcasts, before-trip information (e.g. via Internet), in-vehicle routing and navigation systems).

Traffic management centres (TMCs) are the building blocks for many other AI applications. Travellers can be kept informed

\footnotetext{
* 1 Viliam Lendel, ${ }^{2}$ Lucia Pancikova, ${ }^{2}$ Lukas Falat, ${ }^{3}$ Dusan Marcek

${ }^{1}$ Department of Management Theories, Faculty of Management Science and Informatics, University of Zilina, Slovakia

${ }^{2}$ Department of Macro and Microeconomics, Faculty of Management Science and Informatics, University of Zilina, Slovakia

${ }^{3}$ Research Institute of the IT4Innovations Centre of Excellence, Silesian University in Opava, Czech Republic

E-mail: viliam.lendel@fri.uniza.sk
} 
of possible problems via a personal information assistant or a car navigation system. The most commonly installed devices are VMS and traffic lights. Incident management is an integral part of transport network management and AI techniques are helping to detect, monitor and respond to accidents quickly [4]. Electronic payment and smart cards are already used for payment of public transport fares and road tolls around the world. AI techniques are already widely employed in smart card technology, for example, to combat fraud. The memory and processing capabilities available on smartcard microchips allow development of flexible and innovative products for paying fares and other transport-related charges. Intelligent navigation systems support individual travellers by providing information about the shortest possible routes, the current traffic situation and alternative routes. AI technologies also address the need for dynamic routing depending on information from TMCs and from other travellers. Artificial intelligence can be used in all these applications: arterial management, freeway management, transit management, incident management, emergence management, electronic payment, traveller information, information management, crash prevention and safety, roadway operations and management, road weather management, commercial vehicle operations, and intermodal freight [7].

Artificial intelligence has also an important role in road transport. As for traffic road, the artificial intelligence is used in intelligent traffic signs, in platooning and when calculating the Green Wave (adaptive traffic signal control based on real-time traffic conditions). Artificial intelligence is also used in the car; primarily for managing the accuracy of stopping, braking the car, control air conditioning in the car, anti-collision system, preventing the driver's drowsiness (analysis of eyelid movement of the driver, analysis of variations in the trajectories), vehicle speed control (based on evaluation of road and weather parameters recommended for the maximum permitted speed), control of frequencies in the car windscreen, optimizing fuel consumption, autonomous vehicle control (in the middle of the traffic flow), vision detection and image recognition systems (identification of fixed obstacles, vulnerable road users using sensors and computer processing). Finally, artificial intelligence is used in the field of tunnels. The means of AI check the lighting and ventilation in the tunnel, traffic density assessment, classification of fire in a tunnel under the heat of fire and the fuzzy logic is used for analysis of environmental data (road moisture, road temperature, visibility range...) [8].

The intelligent transport systems can organise and manage transport systems in such way that they can be used as efficiently and economically as possible [9]. Just implementation of appropriate prognostic methods and planning models allows to determine the planned value of supply and demand by evaluation of time series trend introducing the possible solution to reach stable and long-time results of region and transport enterprise activities and also the application of intelligent transport systems [10].

The goal of this paper is to experimentally show the benefit of using a new AI approach in transport problems by applying advanced artificial intelligence methods for time series forecasting. To fulfil this goal, we use the prediction model based on the theory of support vector machines: we find the optimal kernel function as well as type of regression best suited for such data. Authors then perform optimization of SVR (Support Vector Regression) using Open Source Software R in transport management system with affiliation to further research.

The main hypothesis is as follows: by applying new intelligent methods of artificial intelligence into transport area, we can state that there is a clear benefit of using these methods instead of traditional ones. Our approach is novel in using means of artificial intelligence, i.e. support vector regression into transport problems (instead of using standard methods).

The paper is divided into 5 parts. The second chapter introduces traditional (exponential smoothing, ARIMA models, GARCH) as well as intelligent methods (neural networks, support vector machines) in transport. The third chapter discusses the empirical analysis of the Support vector machines; it then deals with describing the basic theory of the SVM regression which is the default model for our prediction model. The fourth chapter discusses the experiment. Finally, chapter five summarizes the paper.

\section{Traditional and intelligent methods in transport}

Predicting processes in intelligent transport systems are realized via different approaches. Quantitative approach, which is one of the most used today, is based on statistical methods. Exponential smoothing [11, 12] was one of the first methods for time series modelling. The method is based on weighting data exponentially based on their age. The method is useful for seasonal and trend data. Kalman filtering [13], i.e. Kalman recursive procedures are another time series technique for forecasting. Kalman is used in many areas. One of them is the modelling seasonal data. It consists of estimating the state variables based on structural models. Box-Jenkins models [14], i.e. ARIMA or SARIMA (seasonal autoregressive integrated moving average) models assume of the dependence of the data in one-dimensional time series. According to the type of the process, these may be suitable either for adjusted, or seasonal time series. $\mathrm{ARCH}, \mathrm{GARCH}$ [15] (generalized autoregressive conditionally heteroscedastic models), or other types of autoregressive conditionally heteroscedastic models are models applicable especially for high frequency data (financial markets), based on failure to meet certain criteria of the autoregressive processes, such as linearity, variance equality and stationarity. Finally, spectral analysis is a time series modelling in the frequency 
domain, suitable for seasonal time series. Unlike other methods, several significant periods (frequencies) can enter the model.

In general, artificial intelligence is inspired by biological processes, generally learning from previous experience. Main tasks for artificial intelligence methods are based on learning from experimental data (including learning from patterns) and transferring human knowledge into analytical models [16]. One of the first machine learning techniques were artificial neural networks (ANN). As ANN was a universal approximator, it was believed that these models could perform tasks like pattern recognition, classification or predictions [17, 18]. In recent years, scientists try to incorporate other factors to increase the accuracy of neural networks, such as Evolving RBF (Radial Basis Function) neural networks in which genetic algorithms are implemented [19]. Even though neural networks are not in the centre of attention, their era has not ended yet. People talk about massive renaissance of neural networks [20]-mainly thanks to publications about deep neural networks [20].

However, in recent years, the flagship of artificial intelligence are support vector machines (SVM) [21,22]. In majority of studies the SVM outperformed artificial neural networks. There are many reasons behind this; one of them is that SVM can find and reach a global minimum. SVM are studies to increase their properties. For example, Cao et al. [23, 24] deal with more effective technique for forecasting using self-organizing maps (SOM).

Moreover, there also exists an AI model which is based on transferring human knowledge (experience) into workable systems and uses fuzzy logic (FL) systems such as in [18].

The application of predictive modelling has exceeded the borders of basic statistics long ago thanks to the current software capabilities. Means of artificial intelligence regularly form a part of commercial and open source software packages. As an example, we can mention support vector machines or certain types of neural networks utilized to solve classification and prediction problems in certain areas of engineering, medicine, economic forecasts, management and transport. The choice of an appropriate prediction method is influenced by the properties of the data, namely its actual extent, the structure of the time series, the monitoring frequency and, in case of causal models, the analysis of the relations between specified quantities. A complex solution of modelling the selected time series comprises of various stages, such as specification, quantification, verification and application. Based on the assessment of the present seasonality a certain way of modelling, based on models having a statistically significant seasonal component and models for adjusted data, is chosen. Comparison of the methods, their combination and subsequent optimisation of the solution is the way to improve the output of predictive modelling.

\section{Suggested SVR prediction model for seasonal data in transport}

Based on the reasons stated in the previous chapter, as well as on base of [25], the authors of this study decided to choose support vector regression as a representative of artificial intelligence technique to test their hypothesis. Another reason why we decided to apply artificial intelligence methods into transport is that artificial intelligence applications are a very efficient method for extremely big data sets (gigabytes of data) where classical statistical or econometrical methods could not be used due to computational or time (cost efficiency) reasons [6]. Finally, we decided to use linearly inseparable model of SVR since linearly inseparable SVR is better choice when forecasting time series instead of cross-sectional data.

\subsection{Methodology}

The Support Vector Machine [26 and 21] has wide application in classification tasks, as well as in predictions (SV regression). SV regression is based on the fundamental principles of the SVM method. The main idea of the SVM regression [27, 22 and 28] (SVR) lies in the search for the optimal band for the regression function. This band can be characterized as an error of the approximation of the actual data by a regression function, thus being the measure of the regression error. It is denoted by e and calculated as:

$e=y_{t}-\hat{y}_{t}$

where $y_{t}$ is the current observed value at the time $t$ and $\hat{y}_{t}$ is the value estimated by the regression function.

Root mean square error is a statistical measure to selection and optimisation of predicted data:

$R M S E=\frac{1}{n} \sum_{t=1}^{n}\left(y_{t}-\hat{y}_{t}\right)^{2}$

Details of the theoretical background for SVM, core functions and linear inseparable data can be found in numerous scientific publications [21, 22]. Current solutions of the classification and prediction options support this method when comparing and evaluating the prediction capabilities and accuracy of this method with conventional statistical methods, whether it is the logistic regression or any of the approaches for modelling. Application of the machine learning methods resources is currently supported by the software applications (e.g. modelling in R) and, compared to the "traditional" statistical methods, it often prevails by having the advantage of raw data input, irrespective of the restrictive assumptions of the approach chosen. 


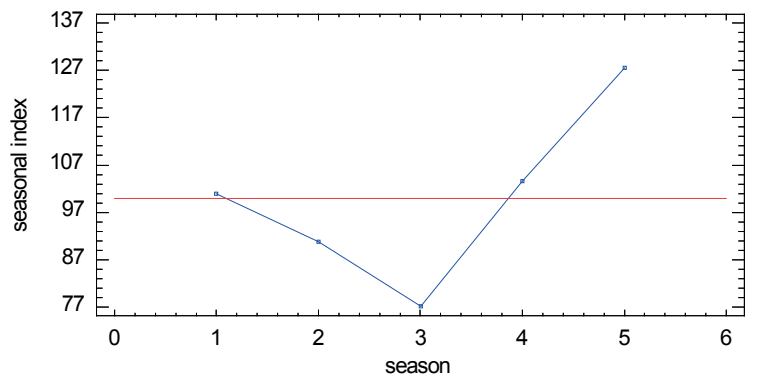

Figure 1 Seasonal indices for selected time series

\subsection{Experiment}

The data we used for our experiments were time series of financial profit of a Slovakian transport company. This company provides the personal bus public transport. The length of the series was 145 weeks. We have chosen to select the optimal (the best) model based on AIC and RMSE, assuming the significance of all model parameters. Therefore, stationarity of time series was not tested. To compare forecasting abilities of two approaches (statistical methods and machine learning methods) we have chosen the daily data of revenues with assumed seasonality. This element was confirmed by the graphical representations of periodogram, autocorrelation and partial autocorrelation function, and season indices which are shown in Figure 1.

As in the text above Figure 1, the presence of seasonality was assessed in several ways. We chose the graphical tools: periodogram, correlograms, and seasonal index chart (Figure 1). For the sake of clarity, this graph has been chosen to show unambiguous fluctuations in the daily work week data (Monday $=1$, Friday $=5$ ).

Both approaches were evaluated using seasonal data as well as cleaned unseasonal data (using moving averages) where the combination with seasonal indices is necessary. Statistical methods were processed in Statgraphics and in R. The methods of SV regression were realized in the R software. We selected the best models based on criteria and RMSE.

As stated above, we chose the R software for our SVR experiments. The detailed instructions on how to use $\mathrm{R}$ for statistical modelling is stated in [29]. To create a SVR (Support Vector Regression) model with open source $R$, we need the package e1071 [30, 31]. We used this package to train a support vector machine. It can be used to carry out general regression and classification (of nu and epsilon-type), as well as density-estimation. Code [32] with default settings to make predictions for selected data with SVR can be seen in [33]. As for $\mathrm{R}$ modelling, environment $\mathrm{R}$ allows the preparation, data processing, quantification and optimisation models based on a combination of selected modelling approaches. R currently offers number of built-in functions in the packages.
This basic SVR setting is not sufficient in many cases, however we can optimize the SVR parameters ("epsilon", "cost") and make further "extensions", such as the choice of kernel function, defining the training and test set. The epsilon parameter reflects the error rate. Valid: if the difference between the actual and estimated value on the regression curve in absolute value is less than or equal to the epsilon, the SVR considers the error to be zero. Constant $C$ penalizes regression function deviations from measured values. To optimize the SVR parameters, we trained more models and then chose the best one.

Based on our R manual [33] we created estimates for selected real-time series. Our SVR models were compared with appropriate statistical models. Table 1 displays some of them.

In our experiments, the centre of attention was at regression models. These models are built on the assumption that there is some sort of dependency in the data. SV regression models bring an appropriate alternative for forecasting seasonal as well as nonseasonal data. The code used to estimate the SVR is as follows

datasvr $2<$-read.csv( “c:/csvtrend.csv", sep=";,, dec=",")

$>$ model $2=\operatorname{svm}(y t 2 \sim$ yt.l, data $=$ datasvr 2, type $="$ eps-regression,

+ kernel="linear", cost $=1$, epsilon $=0.01$ )

$>$ predictions $=$ predict (model2, datasvr 2 )

rmse $2=\operatorname{sqrt}\left(\right.$ mean $\left.\left((\text { datasvr } 2 \$ \text { yt } 2 \text {-predictions })^{\wedge} 2\right)\right)$

$>$ rmse 2

plot(datasvr2\$yt2, type='lines')

lines(predictions, col='red')

$>$ tuneResult $<$ tune $($ svm, yt $2 \sim y t .1$, data $=$ datasvr 2 ,

+ ranges $=\operatorname{list}($ epsilon $=\operatorname{seq}(0,1,0.1)$, cost $=2 \wedge(2: 9))$

+ )

$>$ print(tuneResult)

Figure 2 displays the development of real and estimated values of season-free data using the methods of SV regression. The graph of real values is shown in black, the graph of estimated values is red. The parameters of SVR have been already optimized in R. Figure 3 displays the optimisation output with estimated parameters.

The text and graphical outputs display the results of the "tune“ function. Based on this, they are optimized (their combination) cost and epsilon. The darkest area on the chart shows the best values. The "tune" function optimizes the model based on the RMSE criterion, calculated on the set of data where the model is trained.

\section{Discussion}

Inputs into SVR were independent time lags of the same time series, i.e. the lag of 5 days (seasonal data) and lag of day 1 (season-free data). By optimizing the SVR using the function tune, i.e. finding best values of parameters of 'cost' and 'epsilon', we 
Table 1 Statistical models for seasonal and seasonally adjusted data (source: own experiment performed in R)

\begin{tabular}{cc}
\hline Model - seasonal data & Estimated parameters \\
\hline ARIMA $(0,0,0)(0,1,1)$ & SMA $(1)=0.981$ \\
Seasonal regression model & \\
Holt - Winter expon. smoothing & \\
Model - seasonally adjusted data & $\operatorname{AR}(1)=0.481 ; \operatorname{MA}(1)=-0.402 ;$ \\
& $\operatorname{MA}(2)=-0.424 ; \operatorname{MA}(3)=-0.405 ;$ \\
ARIMA(1, 0, 5) with constant & $\operatorname{MA}(4)=-0.390 ; \operatorname{MA}(5)=0.560 ;$ \\
& $\operatorname{Mean}=66,300$ \\
ARIMA(0, 0, 5) with constant & $\operatorname{MA}(3)=-0.800 ; \operatorname{MA}(4)=-0.700 ;$ \\
$\operatorname{MA}(5)=0.170 ; \operatorname{Mean}=66.300$
\end{tabular}

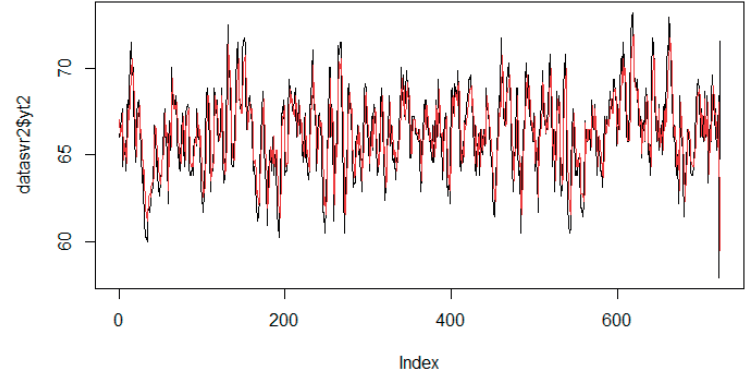

Figure 2 Actual and estimated values with SVR Parameter tuning of 'svm':

- sampling method: 10 - fold cross validation - best parameters: epsilon cost

0.74

- best performance: 2.468643 RMSE $=1.571$

tried to minimize the RMSE error. The best value of RMSE with SVR (season-free data) was RMSE = 1.571 (before optimization the RMSE was equal to1.600).

The lowering of RMSE influences many factors, such as the size of training and validation set, selection of the kernel function (in our experiments we used linear, radial, polynomial kernel functions) and regressors. SVR is a way to go for modelling time series data with a possibility to apply this method in transport management.

\section{Conclusion}

Artificial intelligence has an important place in transport. It can be used in various fields of transport, especially in traffic management systems, incident management, travel information systems, transport management centres and models. Nonlinear models can be used for predicting traffic demand, predicting the

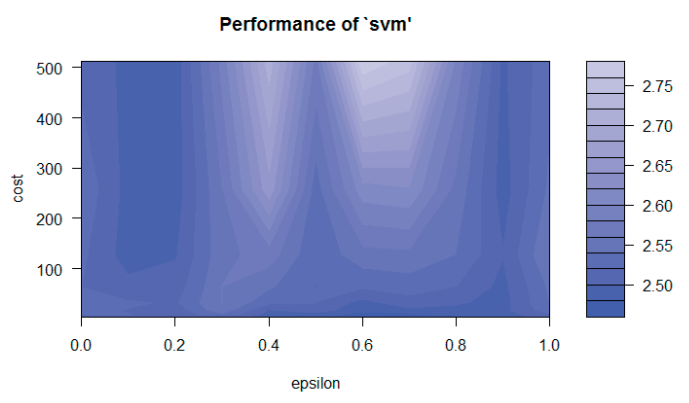

Figure 3 Optimisation of SVM

deterioration of transport. In a transport planning process, AIbased decision support systems can be implemented for transport planning. Optimisation problems based on AI in transport can be solved with designing an optimal transit network for a given community, developing an optimal shipping policy for a company, developing an optimal work plan for maintaining and rehabilitating a pavement network, and developing an optimal timing plan for a group of traffic signals. AI can also be used in controlling a system, i.e. signal control of traffic at road intersections, ramp metering on freeways, dynamic route guidance, positive train control on railroads, and air traffic control. Clustering can be used for the identification of specific classes of drivers based on driver behaviour. Computer-Aided Design can be implemented in the geometric design of highways, interchange design, structural design of pavements and bridges, culvert design, retaining walls design, and guardrail design. Decision making can also benefit from techniques of artificial intelligence; deciding whether to build a new road, how much money should be allocated to the maintaining and rehabilitating activities and which road segments or bridges to maintain, and whether to divert traffic to an alternative route in an incident situation.

In this paper, we firstly discussed the current state in forecasting time series in transport; traditional as well as modern 
AI methods were briefly discussed. Subsequently, we tested the hypothesis that by applying new intelligent methods of artificial intelligence into transport area, we can state that there is a clear benefit of using these methods instead of traditional ones. To test our hypothesis, we performed the empirical analysis of customized SVR prediction model. In our experiment, we used seasonal data of financial profits in personal transport of selected economic subject. By applying the Support vector regression prediction model optimised to given data and by running a comparative analysis, we found out that using AI techniques have a lot to offer to the field of transportation. The versatility of the tools and their performance are well suited for the complexity and variety of transport systems. AI holds promise for a wide range of transport problems, which have been previously approached using other mathematical frameworks. In transport modelling, they are relatively new, but they seem to be a great alternative to traditional models and methods.

\section{Acknowledgement}

This work was partially supported by The Ministry of Education, Youth and Sports from the National Programme of Sustainability (NPU II) project „IT4Innovations excellence in science-LQ1602.

This paper was partially supported by the Slovak scientific grant VEGA 1/0363/14 Innovation management-processes, strategy and performance.

\section{References}

[1] WOSYKA, J., PRIBYL, P.: Decision Trees as a Tool for Real-Time Travel Time Estimation on Highways. CommunicationsScientific Letters of the University of Zilina. 15(2A), 11-16, 2013.

[2] DIZO, J., BLATNICKY, M., SKOCILASOVA, B.: Computational Modelling of the Rail Vehicle Multibody System Including Flexible Bodies. Communications-Scientific Letters of the University of Zilina, 17(3), 2015.

[3] SMITH, K., GUPTA, J.: Neural Networks in Business: Techniques and Application. Idea Group Publishing, Hershey, 2002.

[4] MILES, J. C., WALKER, A. J.: The Potential Application of Artificial Intelligence in Transport. Journal of Intelligent Transport Systems, 153(3), 183-198, 2006.

[5] STENCL, M., LENDEL, V.: Application of Selected Artificial Intelligence Methods in Terms of Transport and Intelligent Transport Systems. Periodica Polytechnica Transportation Engineering, 40(1), 11-16, 2012.

[6] LENDEL, V., STENCL, M.: Possibility of Applying Artificial Intelligence in Terms of Intelligent Transport Systems. Journal of Information, Control and Management systems, 8(1), 37-46, 2010.

[7] RAMOS, C., AUGUSTO, J. C., SHAPIRO, D.: Ambient Intelligence-The Next Step for Artificial Intelligence. Intelligent Systems, 23(2), 15-18, 2008.

[8] SPALEK, J., JANOTA, A., BALAZOVICOVA, M., PRIBYL, P.: DECISION-MAKING and Management with the Support of Artificial Intelligence (in Slovak). EDIS, University of Zilina, p. 374, 2005.

[9] HRKUT, P.: Architecture of Providing Services in Intelligent Transportation Systems Using Semantically Defined Information. Communications-Scientific Letters of the University of Zilina, 12(3A), 75-79, 2010.

[10] DICOVA, J., ONDRUS, J.: Trend of Public Mass Transport Indicators-as a Tool of Transport Management and Development of Regions. Communications-Scientific Letters of the University of Zilina, 12(3A), 121-126, 2010.

[11] BROWN, R. G.: Statistical Forecasting for Inventory Control. McGraw-Hill, New York, 1959.

[12] BROWN, R. G.: Smoothing Forecasting and Prediction of Discrete Time Series. Englewood Cliffs. NJ, Prentice-Hall, 1963.

[13] KALMAN, R. E.: A New Approach to Linear Filtering and Prediction Problems. Transactions of the ASME-Journal of Basic Engineering, 82(D), 35-45, 1960

[14] BOX, G. E. P., JENKINS, G. M.: Time Series Analysis: Forecasting and Control. CA: Holden-Day, San Francisco, 1976.

[15] ENGLE, R. F.: Autoregressive Conditional Heteroskedasticity with Estimates of the Variance of United Kingdom Inflation. Econometrica, 50(4), 987-1008, 1982.

[16] KECMAN, V.: Learning and Soft Computing: Support Vector Machines, Neural Networks, and Fuzzy Logic Models. MIT Press, Cambridge, 2001.

[17] ANDERSON, J. A., ROSENFELD, E.: Neurocomputing: Foundations of Research: A Collection of Articles Summarizing the State-of-the-Art as of 1988. MIT Press, Cambridge, 1988.

[18] HERTZ, J., KROGH, A., PALMER, R. G.: Introduction to the Theory of Neural Computation. Westview Press, Colorado, 1991. 


\section{kom NIKOCle}

[19] RIVAS, V. M., MERELO, J. J., CASTILlO, P. A., ARENAS, M. G., CASTELLANO, J. G.: Evolving RBF Neural Networks for Time-Series Forecasting with EvRBF. Information Sciences, 165, 207-220, 2004.

[20] HINTON, G. E., OSINDERO, S., TEH, Y.W.: A Fast Learning Algorithm for Deep Belief Nets. Neural Computation, 18, 15271554, 2006.

[21] VAPNIK, V.: The Nature of Statistical Learning Theory. Springer-Verlag, New York, 1995.

[22] VAPNIK, V., GOLOWICH, S. E., SMOLA, A.: Support Vector Method for Function Approximation, Regression Estimation and Signal Processing. Advances in Neural Information Processing Systems, 9, 281-287, 1996.

[23] CAO, L. J., TAY, F. E. H.: Support Vector Machine with Adaptive Parameters in Financial Time Series Forecasting. IEEE Transactions on Neural Networks, 14(6), 1506-1518, 2003.

[24] CAO, L. J., TAY F. E. H.: Financial Forecasting Using Support Vector Machines. Neural Computing Applications; 10, 184-92, 2001.

[25] FALAT, L., PANCIKOVA, L., HLINKOVA, M.: Prediction Model for High-Volatile Time Series Based on SVM Regression Approach. Proceedings of IEEE Information and Digital Technologies, Slovakia, 2015.

[26] Support Vector Machines (SVM) Fundamentals Part-I. [online]. Available: https://panthimanshu 17.wordpress.com/2013/07/28/ svm-fundamentals-part-1/ (accessed 12.12.2014).

[27] CAO, D.-Z., PANG, S.-L., BAI, Y.-H.: Forecasting Exchange Rate Using Support Vector Machines. Proceedings of the Fourth International Conference on Machine Learning and Cybernetics, China, 2005.

[28] KIM, K.: Financial Time Series Forecasting Using Support Vector Machines. Neurocomputing, 55, $307-319,2003$.

[29] CRAWLEY, M.: The R Book. Wiley, Chichester, 2007.

[30] CHANG, CH.-CH., Lin, CH.-J.: LIBSVM-A Library for Support Vector Machines [online]. Available: http://www.csie.ntu.edu. tw/ cjlin/libsvm/ (accessed 22.12.2014).

[31] Misc Functions of the Department of Statistics (e1071) [online]. TU Wien. Available: http://cran.r-project.org/web/packages/ e1071/ e1071.pdf (accessed 10.12.2014).

[32] SVM Tutorial [online]. Available: http://www.svm-tutorial.com/2014/10/support-vector-regression-r/ (accessed 10.12.2014).

[33] R - manual [online]. Available: http://fria.fri.uniza.sk/ kmame/drupal/subory/pancikova/Rmanual/ (accessed 11.12.2014). 